DOI: 10.12957/demetra.2015.16173

\title{
Alimentarse o nutrirse en un comedor social en España: reflexiones sobre la comensalidad
}

\section{Feed or nourish in a "popular restaurant" in Spain: reflections on commensality}

Fabiana Bom Kraemer ${ }^{1}$

Mabel Gracia Arnaiz ${ }^{2}$

\section{Universidade do Estado do Rio de Janeiro. Instituto de Nutrição, Departamento de Nutrição Aplicada, Núcleo de Estudos sobre Cultura e Alimentação. Rio de Janeiro-RJ, Brasil \\ ${ }^{2}$ Universidad Rovira i Virgili. Grupo de Investigaciones Antropológicas. Tarragona, Espanha. \\ A primeira versão deste texto foi apresentada na $29^{a}$ Reunião Brasileira de Antropologia, realizada entre os dias 03 e 06 de agosto de 2014, Natal-RN.}

Apoio financeiro: Faperi APQ1 2010/01. CNPq Edital Universal 14/2011. Beca 'doctoradosándwich' FAPERJ 2013, Brasil. Plan Nacional I+D (España - CSO2012-31323).

Correspondência /Correspondence

Fabiana Bom Kraemer

E-mail:fkraemer@uerj.br

\section{Resumen}

Este trabajo señala los cambios en los hábitos alimentarios de las personas en situación de vulnerabilidad social y afectadas por la reciente crisis económica y financiera española. La investigación se realizó en un comedor social en la ciudad de Reus, Cataluña, España. El análisis se refiere específicamente a las observaciones del comportamiento de los comensales en la hora del almuerzo y al uso de las técnicas de grupo focal y entrevistas. Hemos observado que los coordinadores del proyecto valorizan el ritual como una manera de fomentar la solidaridad y la convivencia, dando a la comensalidad un sentido especial de acogida. La recepción es percibida por los comensales a través de la labor de los voluntarios. Sin embargo, los comensales relacionan negativamente la comensalidad con la pérdida de autonomía en la decisión de alimentarse. Cómo, con quién y dónde comer, en el comedor social, no es una opción de los comensales, el acto de comer sigue siendo un interés personal y una satisfacción individual, observándose una individualización del acto de comer durante el tiempo de la comida. "Qué comer" tampoco es una elección del comensal. Para él, el menú se caracteriza por el menor consumo de carne, frutas y verduras y mayor consumo de carbohidratos. A la vez, por la situación de pobreza, hay una resignación frente a la comida que se sirve. El comensal, de esta manera, a pesar de sentirse amparado, muestra una pérdida del referente cultural, de la visión que tiene sobre la calidad nutricional de los alimentos y del poder de tomar decisiones acerca de su comida.

Palabras clave: Comensalidad. Hábitos Alimentarios. Asistencia Alimentaria. Espanha. 


\begin{abstract}
This paper is about the changes in food practices of socially vulnerable people affected by the recent economic and financial crisis in Spain. The fieldwork was conducted in a "popular restaurant" located in Reus, Catalonia, Spain. The analysis refers specifically to observations on commensals' behaviour at lunch time and use of focal groups and interview techniques. The project's coordinators value the ritual meals as a way to promote solidarity and conviviality, giving commensality a special sense of embracement. The "embrace" is perceived by commensals through the volunteer work. However, commensals relate negatively the commensality with loss of autonomy in food decision. As the "who with and where to eat" decision in the restaurant is not on them, the act of eating is a personal interest and an individual satisfaction, observing an individualization of eating during the meal. "What to eat" is also not a commensal choice. For them the menu is characterised by less consumption of meat, fruit and vegetables and higher consumption of "carbohydrates". At the same time, toward poverty, there is a resignation before food is served. Commensals, in spite of feeling supported, present a loss of cultural reference, nutritional quality of food and the power to make decisions about their food.
\end{abstract}

Key words: Commensality. Food Practices. Food Assistance. Espanha.

\title{
Introducción
}

En los últimos años, una serie de cambios sociales han interferido con el comportamiento alimentario en la sociedad occidental, y los debates sobre la alimentación contemporánea han tratado de responder qué consecuencias generan estos cambios, cómo suceden y en qué dirección están caminando.

Los estudios sociológicos y antropológicos ubican en la llamada "modernidad alimentaria" los impactos que ha sufrido la comida, por las transformaciones sociales, económicas y culturales en la sociedad contemporánea. Por una parte, se caracteriza por la abundancia en el suministro de alimentos, el aumento de la producción y el consumo de alimentos procesados, el aumento de los conocimientos científicos acerca de la composición de los alimentos y la multiplicación de los discursos y las normas dietéticas. Por otra, estos cambios han impuesto a los comensales la 
desorganización en los modos de comer, la homogeneización de lo que se come, la mala nutrición y la inseguridad respecto a los componentes de los alimentos. ${ }^{1-3}$

Estudios españoles que establecen el nexo causal entre el proceso social de la modernización de la sociedad y los cambios alimentarios demuestran la tendencia a una nueva estructura de la conducta alimentaria, con una simplificación de la estructura alimentaria (que en España sería un solo plato y postre), un cambio en el horario y en el tipo de comidas que se hacen por las actividades laborales y escolares y la medicalización de la alimentación.--6

Gracia Arnaiz ${ }^{6}$ nos recuerda que, en los países industrializados, la mayor accesibilidad a los alimentos contrasta con la persistencia del consumo diferencial y socialmente desigual, teniéndose en cuenta el nivel de ingresos de las personas, lo que indica que para muchas personas los problemas de la modernidad alimentaria no son los de sobreabundancia. Datos de Food and Agriculture Organization of the United Nations informan que alrededor de 842 millones de personas en todo el mundo padecieron hambre crónica en el período comprendido entre 2011 y 2013. ${ }^{7}$ Por lo tanto, parece que la democratización del consumo de alimentos no está siendo real y todavía existen diferencias dietéticas relacionadas con variables socioeconómicas.

En este sentido, tratamos de comprender los efectos de los cambios macro-sociales en la dieta, teniendo por referencia los efectos de la adopción de una política económica neoliberal - la globalización y los procesos de cronicidad del desempleo, la miseria y la pobreza por ella favorecidas. ${ }^{8}$ Así, este trabajo sitúa los cambios en los hábitos alimentarios diarios de personas en situación de vulnerabilidad social y afectadas por la crisis económica y financiera reconocida oficialmente en 2008 en España.

España ha demostrado desde entonces un gran número de personas en la pobreza temporal o recurrente ${ }^{9}$ que se dirigen a la cronicidad. Según datos de Caritas Española, ${ }^{10}$ el $58 \%$ de los españoles son parados de larga duración (que están sin trabajo en los últimos 12 meses) y el 35\% están desempleados desde hace más de dos años, y también una de cada tres personas asistidas por la entidad demanda ayuda hace más de tres años.

En este escenario, hemos observado un aumento de la ayuda alimentaria a los "nuevos pobres" por el gobierno, instituciones no gubernamentales y la sociedad civil, con, entre ellos, la distribución de comidas en comedores sociales.

Estas "nuevas" formas de comer (que parten de filantropías) cargan enellas cambios en el comportamiento, en las representaciones y percepciones sociales relacionadas con la alimentación y necesitan ser mejor comprendidas y analizadas. En este sentido, queremos saber en qué medida la crisis financiera y económica convierte las maneras de comer de las personas en precarias condiciones de vida y contribuyen al debate sobre las paradojas y ambigüedades del sistema alimentario actual. 


\section{Consideraciones Metodológicas}

Un estudio se ha diseñado para la reflexión sociocultural, ${ }^{(*)}$ con el fin de abordar la relación entre los cambios en los hábitos alimentarios y el progresivo empobrecimiento de una parte de la población española. Así que optamos por un enfoque cualitativo de investigación desde la perspectiva socio-antropológica.

El trabajo de campo se llevó a cabo en un comedor social creado a finales de 2009 de una asociación entre una agencia gubernamental y una institución no gubernamental (católica) en la ciudad de Reus, Cataluña, España. El enfoque es etnográfico y recurrimos a las técnicas de investigación, tales como la observación directa, entrevistas en profundidad, grupos focales y el uso del diario de campo. ${ }^{11,12}$

En la medida en que estamos interesados en conocer y comprender los cambios en las formas de comer de los afectados por la crisis económica y financiera en España, nuestra investigación buscó privilegiar el punto de vista de los diferentes actores sociales. Por lo tanto, nos interesó la incorporación de las experiencias de los que viven en situaciones críticas y requieren ayuda alimentaria para que se alimenten, así como los que trabajan en el servicio de asistencia social o colaboran con él.

En principio, hemos desarrollado un script observacional ${ }^{16}$ por lo cual buscamos identificar la dinámica de las relaciones entre las personas, con el espacio y la comida durante las comidas en el comedor. Para la entrevista y el grupo focal se ha buscado formular preguntas acerca de la gestión del comedor social, los comensales, las razones por las que los comensales empezaron a comer en el comedor, la alimentación de los comensales y la crisis en España.

Así, el análisis se refiere específicamente a las observaciones del comportamiento de los comensales durante el almuerzo en un espacio social definido por un grupo de focal con 5 comensales y también 5 entrevistas: una con la trabajadora social de la institución no gubernamental, una con la coordinadora del comedor social y 3 con los comensales.

En la dimensión de este artículo sólo se incluye la discusión de temas relacionados con la comensalidad en torno a una comida diaria en un contexto precario. Por lo tanto, con el fin de ayudar en la reflexión de los efectos de los cambios sociales en los patrones de alimentación de un grupo particular, el análisis fue en las relaciones que se producen alrededor del modo de "comer".

(*) La investigación forma parte del proyecto "Comer en tiempos de crisis: nuevos conceptos alimentarios y de salud en España” desarrollado en la Universitat Rovira i Virgili (Tarragona, ES) y se realizó durante la práctica del doctorado en el exterior para concluir parte del proyecto de tesis "Sentidos e significados da alimentação saudável”: Restaurantes populares no Brasil y comedores sociales na Espanha" (Programa de Posgrado en Nutrición, Alimentación y Salud de la Universidade do Estado do Rio de Janeiro, Brasil). 
Consideramos que la noción de comensalidad en sus diversas dimensiones. Si por un lado, "comer en la misma mesa" crea lazos y establece la sociabilidad, por otro, no es sin dolor y tiene efectos de exclusión. ${ }^{17}$

El comedor social es un programa de distribución de alimentos que cumple la función de dar la comida, libre de pagos o con una pequeña contribución, a la gente de escasos recursos. Así, da la atención a los residentes de la ciudad en riesgo de exclusión social que no pueden cubrir por sí mismos las necesidades del alimento; entre ellos, están las personas directamente afectadas por la crisis económica a través de la pérdida de empleo, los ancianos, los drogadictos y los que tienen enfermedades mentales. Casi todos hombres.

En la ciudad, a través del comedor social, se distribuía, de lunes a viernes, el almuerzo para un grupo de 50 a 60 comensales y se distribuían, todavía, bolsas con alimentos listos para el consumo en los fines de semana con el fin de gestionar de forma más eficiente la escasez a la alimentación. Las comidas de almuerzo son entre trece y catorce horas con treinta minutos.

El almuerzo prácticamente se prepara con los productos alimenticios procedentes de donaciones (banco de alimentos y donaciones privadas), así como la organización de las bolsas para los fines de semana. Por eso, la cocinera y la coordinadora de los menús del comedor social los planean con géneros que estén disponibles.

Se dejó claro a los participantes que la participación estaba libre y, si fuera necesario, habrían que firmar la Carta de Consentimiento.

\section{El comer en el comedor social: formas y significados de la comida}

Es la base de la socio-antropología de la alimentación entender las prácticas sociales, económicas y simbólicas relacionadas con la comida, entendida como un acto social y cultural y, por tanto, productora de diversos sistemas alimentarios. En la creación de estos sistemas, intervienen factores ecológicos, históricos, culturales, sociales y económicos que implican representaciones e imaginarios sociales, involucrando elecciones y clasificaciones. ${ }^{18}$ Así que el hombre, al alimentarse, crea prácticas y asigna significados a lo que está incorporando a si mismo, lo que va más allá de la utilización de los alimentos por el cuerpo.$^{19}$

$\mathrm{O}$ alimentar-se assume sentidos que dizem respeito à significação no contexto de um universo imaginário e simbólico, não necessariamente racional [mas] capaz de produzir identidades individuais e coletivas, relações sociais e vínculos que ultrapassam a lógica consciente do discurso. ${ }^{20}$ 
Sentidos y significados se atribuyen, por lo tanto, a la alimentación, directamente relacionados con el sentido de nosotros mismos como órgano sustantivo de materiales culturales históricamente derivados. ${ }^{21}$ Por lo tanto, lo que se come no puede ser comido también por otra persona, ${ }^{22}$ lo que hace que el acto de alimentarse pueda tener diferentes significados entre los sujetos en el mismo espacio social.

El "comer juntos" adquiere muchos significados, ritual y simbólico, muy superior a la mera función biológica. Esta forma de compartir, intercambiar y reconocer se llama comensalidad ${ }^{23}$ y la pluralidad de significados que posee tiene que ver con los diferentes estratos sociales y las situaciones existenciales que ocurren. ${ }^{24}$

El "comer simbólico" comprende la ingesta de valores vinculados a los alimentos y se relaciona con el valor simbólico de los alimentos consumidos en de comúny con el enlace simbólico de la comida en grupo. Tal que las primeras formas de comensalidad establece rápidamente la relación entre las reglas de intercambio, la estructura de los grupos y la afirmación de identidad. La noción de comensalidad reúne las características de la hospitalidad y de la mesa y tiene un fuerte vínculo con la el acto de convivir, no resumiéndose en el comer, sino también en ser visto comer y saber comer en común. ${ }^{23}$

Los estudios sobre la comensalidad, desde sus primeros textos, se ocupan de fiestas y banquetes, entretenimiento, ritos de diferentes poblaciones y / o las ocasiones, pero pocos en los sitios de comensalidad popular.

En este estudio, hemos tratado de trabajar con grupos sociales "nuevos" que aparecen en este caso en particular, en España, debido a la crisis económica mundial aguda. Con una economía estancada, la tasa de pobreza en este país se eleva a tres millones de personas y las nuevas formas de pobreza se presentan de variadas maneras, que Gracia-Arnaiz ${ }^{25}$ designa como precarización: las familias con adultos desempleados, inmigrantes ilegales, personas mayores con pensiones pequeñas, familias de clase media, debilitados por la falta de recursos, etc. Este empobrecimiento lleva consigo cambios en la forma de comer que se traducen en diferentes opciones de alimentos que van desde la mayor adquisición de las llamadas marcas blancas, ${ }^{(*)}$ prioridad al precio del producto como criterios de elección, creación de huertas hasta la búsqueda por ayudas de atención, entre otros.

Por lo tanto, los reflejos en la comensalidad también se pueden presentar en diferentes formas de acuerdo con las experiencias. Así, en el comedor social, las formas y significados que trae la comida a aquellos que proporcionan asistencia en el comedor se presentan de manera diferente en comparación a los que reciben la ayuda - los comensales. El grupo que trabaja de forma directa o indirecta en el comedor social está directamente relacionado con el gran valor social atribuido, en

(**) Es la marca de productos que pertenece a una cadena de distribución con la que se venden productos de distintos fabricantes. Conocida también como marca propia o genérica. 
casi todas las sociedades, a la realización de una comida común, reforzada por la simbología que la comida tiene para la iglesia católica, que lleva la comida como un fuerte símbolo de la comunión.

El espacio del comedor social en la ciudad de Reus se gestiona a través de una organización católica y, en el sentido religioso, la comida es de fundamental importancia, es decir, comer da la vida y fortalece el alma. En esta perspectiva, la participación de varias personas en la misma comida es una práctica de la comunión abierta al perdón, a la hospitalidad y a la amistad. ${ }^{26}$ Consumir como comensal es estar en comunión con los demás que con nosotros comen. ${ }^{27}$

Así que, el ritual de las comidas diarias se valoriza como una manera de fomentar la solidaridad y la convivencia. En este sentido, se utiliza el acto de comer como vehículo de relación social, enseñando la comensalidad rasgos de hospitalidad en un sentido determinado especial de acogida. Dice uno de los entrevistados: "No es simplemente el hecho de dar comida. Se hace un poco de acogida". La comida conjunta tiene éxito en conseguir un gran valor social en esta perspectiva y las reglas de la comida y la bebida se forman, como la regularidad y la jerarquía de las comidas.

A comensalidade conduz igualmente à regularidade na hora de comer, pois um círculo de pessoas apenas poderia se encontrar em horas predeterminadas - esta foi a primeira superação do naturalismo do ato de comer. Segue-se, na mesma direção, o que se poderia chamar de hierarquia da refeição: não se tira mais da gamela, ao bel prazer e sem regras, mas, para se servir, passa-se a respeitar uma certa seqüência. Nos clubes de comerciantes ingleses, precursores das associações sindicais de hoje, havia uma multa para quem bebesse fora da sua vez. ${ }^{22}$

De esta manera, la comida se consume por todos al mismo tiempo con las características del menú tradicional de España (el tipo ternario, presentado en primer plato, segundo plato y postre) y económico. La comida se sirve directamente en la mesa por voluntarios donde los comensales se reúnen en grupos de quatro personas. La mesa, según lo descripto por Fernandes, ${ }^{24}$ se utiliza como una manera de hacer el intercambio de estima social, porque a través de la referencia de familia promueve el acercamiento entre las personas, es un rito de agregación y la comida como el espacio y el tiempo de participación conduce a un momento de sociabilidad. Así, se trata de proporcionar, a través del ritual de la comensalidad una socialización que permite que los comensales no caigan en el vacío de la vida cotidiana y contribuyan a superar el sufrimiento derivado de los determinantes socioeconómicos que pesan sobre ellos.

En estas comidas, se afirma la importancia de la acogida, porque además del mantenimiento de la estructura de la comida, la selección de alimentos que entran en la preparación de los platos y técnicas culinarias utilizadas son propias de la identidad alimentaria española, aunque haya la presencia de inmigrantes. Por eso, forman parte del menú preparaciones como fideo, paella, estofado de carne etc. que remiten, a la memoria del grupo, los saberes alimentarios preferidos por esa sociedad. 
Esto no significa necesariamente que se cumplan las expectativas y deseos de los comensales. La acogida se percibe por los comensales a través de la labor de los voluntarios que, de acuerdo a los comensales, son siempre muy amables y pacientes. Sin embargo, los comensales relacionan negativamente la comensalidad con la pérdida de autonomía en la decisión alimentaria, lo que nos lleva a la evidencia de las desigualdades sociales, ya que se sobresalen a través de los alimentos, a través de las opciones del tipo y de la forma por que se sirven.

Los elementos de distinción atraviesan los siglos y siguen en vigor. Desde la Edad Media, la comida y las refecciones son símbolos de riqueza y poder, por lo tanto, distinguen estratos sociales. La alimentación de los campesinos ricos, por ejemplo, era más abundante y diversa que la de los pobres. La carne "buena" estaba reservada para las clases sociales más altas, mientras que la peor calidad fue pensada para satisfacer las necesidades de los más desprovidos. ${ }^{28}$

De hecho, los comensales tienen poca (o ninguna) autonomía para decidir "qué", "dónde", "cuándo" y "con quién" comer. Más que alimentarse de acuerdo con el medio que se inserta el hombre, él se alimenta de acuerdo con la sociedad a la que pertenece y, para muchos, la estructura socio-económica actual del país y la falta de lazos familiares hacen que sea esencial hacer la refección en el comedor social.

La casa o el restaurante comercial se señalaron como espacios ideales en referencias alimentarias de los comensales, puesto que allí se come lo que uno quiere (tipo de alimento y el método de preparación) y se puede elegir “con quién” se come.

A pesar de la preparación de platos culinarios cercanos al diario español, "qué" comer no es una elección del comensal. Muchos sólo hacen una comida durante el día y para ellos, la comida es insuficiente, la especia es diferente y lo que les dan es solo para satisfacer el hambre, porque el menú se caracteriza por un menor consumo de carne, frutas y verduras y un mayor consumo carbohidratos. "No nos dan gambas, ni langosta. [...] Todo lo que nos dan es para cebarnos. "(comensal 1). La bolsa de comida que reciben los fines de semana no se considera suficiente y apropiada para preparar una refección, porque se trata de alimentos para pronto consumo, como una barra de pan, jugo o leche y "chucherías" (caramelo, chocolate, galletas). Hay algunas veces que ponen una lata de atún, sándwiches; no hay huevos, aceite de oliva, etc. De tal manera que asocian los alimentos que reciben en la bolsa a la "comida de niños", principalmente, por contener golosinas.

Podemos ver que para los comensales el acto de comer a la mesa en el comedor social radica en la actitud del propósito de subsistencia, una realidad inherente a la estructura misma del hombre y de la condición en la que se encuentran. Al alimento se instituyó su función de nutrir. La comida también marca la distinción social entre "pobres" y "ricos", (re)posicionando los comensales, a través de la escasez y del tipo de alimento, como pobres y dependientes de la solidaridad social. 
No solo "qué" comer, sino también " con quién” comer no es una elección de los comensales. La referencia al comportamiento de drogadictos (hablar en un tono voz más alto o verbalmente agredir a otro comensalo voluntario) por los otros comensales es motivo de incomodidad y aparece como un indicador de la diferenciación social. Las reglas de comportamiento a la mesa, que se define en todas las sociedades, son esenciales para mantener la armonía en las comidas, en la constitución de la civilidad y cortesía. Al ser violadas, se rompe la armonía de las relaciones sociales en el espacio y debilita el vigor socializador de la comensalidad.

Las sociabilidades, organizadas en comedor social, están condicionadas no por la convergencia de los intereses de los sujetos, sino por las condiciones en común de la precariedad en la que se encuentran. La convivencia y la sociabilidad, de esta manera, están en peligro debido a que dependen del proceso interactivo entre el grupo, mediadas por narrativas particulares desarrolladas en el acto comunicativo basado en afinidades.

El elemento de socialización abandona. En cierto modo, en el comedor social, así como informa Simmel ${ }^{22}$ sobre el table d'hôte,

[...] as pessoas se encontram apenas e dominantemente pela razão de comer; estar junto de alguém não é a situação desejada com um valor em si, mas, ao contrário, apesar de estar compartilhando uma mesa com todas essas pessoas, de modo algum se pressupõe que se entre em relacionamento com elas. Todas as baixelas ricas de mesa, como também todo bom comportamento, não podem remediar a situação posta pela finalidade materialista de comer: a aversão demonstrada pela sensibilidade refinada contra a table d'hôte demonstra que apenas a socialização pode canalizar aquela finalidade a uma ordem estética mais elevada. Os atrativos dessa ordem não funcionam quando a situação de estar junto como tal não tem nenhum sentido próprio, quando, em certo sentido, falta a alma, e as baixelas e louças não podem esconder a situação de constrangimento, nem mesmo a feiúra do ato físico de comer.

La afinidad que converge alrededor de la mesa del comedor social, el lazo de la crisis que los une, no trae beneficio a la conversación y observamos, entonces, entre muchos, cierto aislamiento en el acto de comer, a pesar de que comparten la misma mesa y espacio. Cómo o con quién y dónde se come no es una elección de los comensales, el acto de comer permanece como interés y satisfacción individuales.

La comensalidad, que a menudo tiene el significado central en el juego de las interacciones sociales, en el comedor social, se vuelve mucho más en un lugar de nutrirse que de convivencia social. 
Por último, a pesar de los aspectos negativos relacionados por los comensales a la comensalidad, observamos cierta conformidad con lo que les está servido, pues que en situación de pobreza uno debe aceptar lo que le es dado. Los comensales entienden el beneficio de la asistencia como ayuda alimentaria y no como violencia que se inflige a su derecho a la alimentación. Por sentirse impotente ante el impacto de la situación macroeconómica en sus vidas, se silencian a sí mismos.

\section{Consideraciones finales}

En este estudio se busca contribuir a los debates en torno a las paradojas del sistema alimentario moderno a partir del análisis de las formas de comer de las personas que fueron directamente afectadas por la crisis financiera y económica que afecta a España en los últimos años.

Con el aumento del desempleo en el país, la necesidad de buscar la ayuda alimentaria se convierte en una realidad para muchos españoles. Entre las asistencias alimentarias están los comedores sociales, que entregan comidas a personas que no son capaces de proporcionar sus propios alimentos.

En el comedor social de la ciudad de Reus (Cataluña) se busca dar a la comensalidad un espacio acogedor. Sin embargo, se sabe que el acto de comer está imbuido de significados diferentes de acuerdo con el actor social y el contexto.

El comer, para el comensal del comedor social, se convierte en mera operación de subsistencia con renuncia al placer que está en el sabor de la comida y en la elección de "qué" y "cuánto" comer. El comensal, de esta manera, a pesar de sentirse apoyado, muestra la pérdida de la referencia cultural y del poder para tomar decisiones sobre su comida. También considera la pérdida de calidad nutritiva de los alimentos, incluyendo la reducción de la diversidad de la dieta y de la cantidad de comida.

La sociabilidad existente, para varios autores, alrededor de la mesa se debilita a la medida que los comensales no eligen "con quién" comen y la individualización del comer es frecuente, aunque sea en la comida compartida.

Estas reflexiones nos hacen (re)pensar sobre algunas tendencias positivas atribuidas al sistema alimentario moderno, como la democratización de la alimentación social y la reducción de las diferencias sociales en el consumo de alimentos. 


\section{Referencias}

1. Fischler C. El (h)omnívoro: el gusto, la cocina, el cuerpo. Barcelona: Anamgrama; 1995. 421p.

2. Poulain JP. Sociologias da alimentação: os comedores e o espaço social alimentar. Florianópolis: UFSC; 2004. 310p.

3. Contreras J, Gracia M. Alimentação, sociedade e cultura. Rio de Janeiro: Editora Fiocruz; 2011. 496p.

4. Contreras J. La modemidat alimentaria: entre la desestructuració i laproliferació de codis. In: J. Contreras J, coordenador. La vida quotidiana a troves deis segles. Barcelona: 2002. p. 349-363.

5. Díaz Méndez C. Cambios en el consumo alimentario en España: algunas propuestas de análisis de los comportamientos alimentarios. Abaco: Revista de Cultura y Ciencias Sociales 2002; 31:57-72.

6. Gracia Arnaiz M. Em direção a uma nova ordem alimentar? In: Canesqui AM, Garcia RWD, organizadoras. Antropologia e nutrição: um diálogo possível. Rio de Janeiro: Editora Fiocruz; 2005. p. $147-164$.

7. Food and Agriculture Organization. The state of food and agriculture: food systems for better nutrition. Rome: FAO; 2013.

8. Santos M. Por uma outra globalização: do pensamento único à consciência universal. 13a ed. Rio de Janeiro: Record; 2006. 174p.

9. Eurostat. European Commission. Expenditure on social protection [Internet]. 2013. [acesso em: 20 nov. 2013]. Disponível em: http://epp.eurostat.ec.europa.eu/portal/page/portal/social_protection/ data/main_tables

10. Cáritas Espanhola. VIII Informe del Observatorio de la Realidad Social. 2013. [acesso em: 21 nov. 2013]. Disponível em: http://www.caritas.es/publicaciones_compra.aspx?Id=4706\&Diocesis=1\&I dioma $=1$.

11. Nunes EO. A aventura sociológica. Rio de Janeiro: Zahar; 1978. 331p.

12. Costa AF. A pesquisa de terreno em sociologia. In: Silva AS, Pinto JM. Metodologia das ciências sociais. 12a ed. Porto: Afrontamento; 2003. p. 129-148.

13. Flick U. Introdução à pesquisa qualitativa. $3^{\text {a }}$. ed. Porto Alegre: Artmed; 2009.

14. Fontana A, Frey JH. The interview: from structured questions to negotiated text. In: Denzin NK, Lincoln YS, organizadores. Handbook of qualitative research. London: Sage Publications Inc; 2000. p. 645-672.

15. Trad LAB. Grupos focais: conceitos, procedimentos e reflexões baseadas em experiências com o uso da técnica em pesquisas de saúde. Physis 19(3):777-796.

16. Fernandes FMB. Considerações metodológicas sobre a técnica da observação participante. In: Mattos RA, Baptista TWF. Caminhos para análise das políticas de saúde. Rio de Janeiro: IMS/ENSP; 2011. p. 262-274. [acesso em: 30 ago. 2011]. Disponível em: http://www.ims.uerj.br/ccaps/wp-content/ uploads/2011/10/LivroCompleto-versao-online.pdf 
17. Fischler C. Commensality, society and culture. Social Science Information 2011; 50(3-4):528-548.

18. Canesqui AM, Garcia RWD. Uma introdução à reflexão sobre a abordagem sociocultural da alimentação. In: Canesqui AM; Garcia RWD, organizadoras. Antropologia e nutrição: um diálogo possível. Rio de Janeiro: Editora Fiocruz; 2005. p. 9-19.

19. Maciel ME. Cultura e alimentação ou o que têm a ver os macaquinhos de Koshima com BrillatSavarin? Horiz. Antropol. 2001; 7(16):145-156.

20. Carvalho MCVS, Luz MT, Prado SD. Comer, alimentar e nutrir: categorias analíticas instrumentais no campo da pesquisa científica. Ciênc. Saúde Coletiva 2011; 16(1):155-163.

21. Mintz SW. Comida e antropologia: uma breve revisão. Revista Brasileira de Ciências Sociais 2001; 16(47):31-41.

22. Simmel G. Sociologia da refeição. Estudos Históricos 2004; 1(33):159-166.

23. Boutaud JJ. Comensalidade: compartilhar a mesa. In: Montandon A, organizador. O livro da hospitalidade: a acolhida do estrangeiro na história e nas culturas. São Paulo: Editora Senac; 2011. p. $1213-1230$.

24. Fernandes AT. Ritualização da comensalidade. Sociologia: Revista da Faculdade de Letras do Porto 1997; 7(8):7-30.

25. Gracia Arnaiz M. Comer em tempos de 'crisis': nuevos contextos alimentarios y de salud em España. Salud Pública de México 2014; 56(6):648-553.

26. Teixeira C, Silva AWC. A cultura da mesa de refeição e o seu aspecto teológico religioso. Revista Eletrônica Espaço Teológico [Internet] 2013; 7(11):02-11. [acesso em: 03 jun. 2014]. Disponível em: http://revistas.pucsp.br/index.php/reveleteo/article/view/15685

27. Boff L. Comensalidade: passagem do animal ao humano. Jornal do Brasil, 14 out. 2012. Colunista. [acesso em: 21 abr. 2014]. Disponível em: http://www.jb.com.br/leonardo-boff/noticias/2012/10/14/ comensalidade-passagem-do-animal-ao-humano/

28. Rodrigues HAF. Alimentação como fonte de sociabilidade e de hospitalidade. SINAIS - Revista Eletrônica. Ciências Sociais [Internet] 2012; 1(12):85-100.

Recebido: 25/4/2015

Aprovado: 28/5/2015

\footnotetext{
APOIO

O artigo é derivado da tese de doutorado "Significados da alimentação em programas de distribuição de refeições: o caso dos restaurantes populares no Brasil e dos comedores sociales na Espanha." Fabiana Bom Kraemer. - 2014, apresentada como requisito parcial para obtenção do título de Doutor, ao Programa de PósGraduação em Alimentação, Nutrição e Saúde, da Universidade do Estado do Rio de Janeiro. Apoio financeiro como Coordenador do Projeto Concepções de alimentação saudável na sociedade contemporânea: reflexões a partir das Ciências Humanas e Sociais. FAPERJ APQ1 2010/01. CNPq Edital Universal 14/2011. Bolsa modalidade doutorado-sanduiche FAPERJ 2013. Na Espanha fez parte do projeto 'Comer en tiempos de crisis: nuevos contextos alimentarios y de salud en España' desenvolvido na Universitat Rovira $i$ Virgili. Plan Nacional I+D (España - CSO2012-31323)
} 